\title{
Identification en aveugle des paramètres de systèmes non linéaires
}

\author{
Sébastien lagrange ${ }^{1}$, Luc Jaulin ${ }^{2}$, Christian Jutten ${ }^{3}$, Vincent Vigneron ${ }^{3}$ \\ 1 LISA \\ $2 E 3 I 2$ \\ 3 LIS \\ Université d'Angers - ISTIA \\ ENSIETA \\ INPG - UJF \\ 62 av. Notre Dame du Lac \\ 2 rue F. Verny \\ 46 Avenue F. Viallet \\ 49000 Angers \\ 29806 BREST \\ 38000 Grenoble \\ lagrange@istia.univ-angers.fr \\ jaulin@ensieta.fr jutten@inpg.fr
}

RÉSUMÉ. Cet article traite du problème d'estimation des paramètres de systèmes non linéaires à temps continus pour lesquels les signaux d'entrées ne sont pas mesurés. L'objectif est de montrer qu'uniquement à partir d'hypothèses statistiques faibles sur les entrées inconnues (telle l'indépendance), il est possible d'identifier les paramètres inconnus d'un système.

Tout d'abord, nous formalisons cette notion sous le nom d'identifiabilité en aveugle par analogie avec l'identifiabilité au sens classique (lorsque les entrées et sorties sont connues). Des conditions nécessaires et suffisantes d'identifiabilité sont introduites et illustrées sur des exemples. Ensuite, nous présentons une méthodologie de résolution de ce problème d'estimation paramétrique en aveugle ainsi que des simulations. A noter que l'originalité de la méthode d'estimation en aveugle proposée repose sur l'utilisation conjointe de résultats sur les dérivées des signaux aléatoires et des techniques de résolution d'analyse par intervalles qui ont l'avantage d'être globale et garantie.

ABSTRACT. This paper deals with the parameter estimation problem of nonlinear systems when the input signals are not measured. The aim is to show that it is possible to identify the system parameters only from weak statistical assumptions on the unknown inputs (such independency). A formalization of this concept, call blind identifiability, is introduced by analogy with identifiability (when the inputs and outputs are known).

Finally, an illustration of these results is given considering various examples and simulations in the particular case of the unknown inputs are supposed to be independent. It should be added that the originality of the blind estimation method proposed is based on results concerning random signal derivatives with interval analysis techniques which remain guaranteed.

MOTS-CLÉS : Estimation en aveugle, Identifiabilité en aveugle, dérivation de signaux aléatoires. KEYWORDS: Blind estimation, blind identifiability, random signal differentiation. 


\section{Introduction}

Dans ce papier, nous nous intéressons à la classe des modèles paramétrés décrient par des systèmes inversibles, c'est-à-dire où les entrées dépendent des sorties et de leurs dérivées jusqu'à un ordre fini. Formellement, un système inversible paramétré de degré relatif $r$ s'écrit sous la forme

$$
\mathbf{u}(t)=\psi\left(\mathbf{p}, \mathbf{y}(t), \dot{\mathbf{y}}(t), \ldots, \mathbf{y}^{(r-1)}(t), \mathbf{y}^{(r)}(t)\right)
$$

où $t \in \mathbb{R}$ est le temps, $\mathbf{u}(t) \in\left(\mathbb{R}^{\mathbb{R}}\right)^{n}$ est le vecteur des $n$ entrées, $\mathbf{y}^{(i)}(t) \in\left(\mathbb{R}^{\mathbb{R}}\right)^{n}$ est la $i$-ième dérivée du vecteur de sorties $\mathbf{y}(t), \mathbf{p} \in \mathcal{P}=\mathbb{R}^{n_{p}}$ est le vecteur des $n_{p}$ paramètres et $\psi: \mathcal{P} \times\left(\mathbb{R}^{\mathbb{R}}\right)^{n(r+1)} \rightarrow\left(\mathbb{R}^{\mathbb{R}}\right)^{n}$ est une fonction analytique. Ici, $\mathbb{R}^{\mathbb{R}}$ désigne l'ensemble des signaux définis de $\mathbb{R}$ à valeur dans $\mathbb{R}$.

Remarque 1 Tous les systèmes plats introduits par Fliess dans [FLI 95] sont des systèmes inversibles. Ainsi, la classe des systèmes inversibles modélisent de nombreux systèmes physiques.

Exemple 1 Considèrons le système de la Figure 1 costitué de deux bacs d'eau. Les entrées $u_{1}$ et $u_{2}$ sont les débits d'eau d'entrant dans chaque bac et les sorties $y_{1}$ et $y_{2}$ sont les hauteurs d'eau des deux bacs. Les paramètres a et b sont respectivement la section du canal qui relit les deux bacs et la section des canaux de fuite aux extrémités de chaque bac.

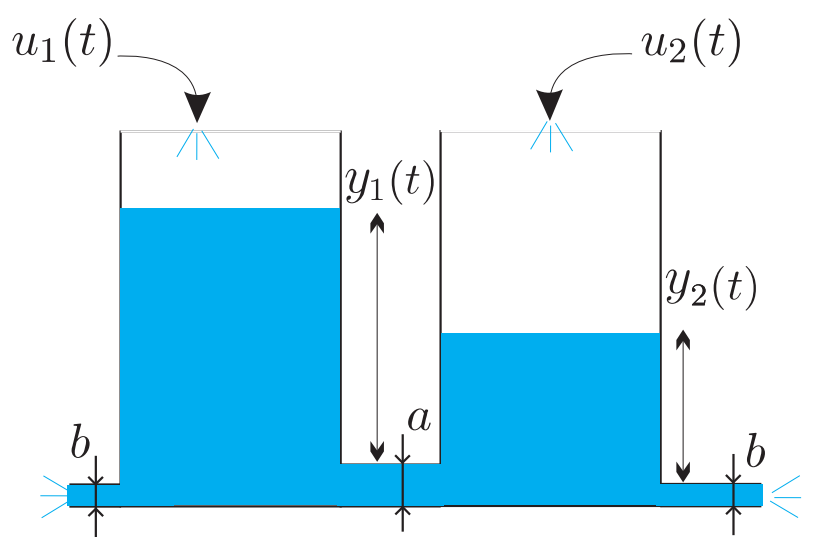

Figure 1. Système hydraulique formé de deux bacs remplis d'eau reliés par un canal.

Ce système régi par les équations d'états suivantes [JAU 05]

$$
\left\{\begin{array}{l}
\dot{y}_{1}(t)=-b \sqrt{2 g y_{1}(t)}-a \cdot \operatorname{sign}\left(y_{1}(t)-y_{2}(t)\right) \sqrt{2 g\left|y_{1}(t)-y_{2}(t)\right|}+u_{1}(t) \\
\dot{y}_{2}(t)=-b \sqrt{2 g y_{2}(t)}+a \cdot \operatorname{sign}\left(y_{1}(t)-y_{2}(t)\right) \sqrt{2 g\left|y_{1}(t)-y_{2}(t)\right|}+u_{2}(t)
\end{array}\right.
$$


s'écrit également sous la forme

$$
\mathbf{u}(t)=\psi(\mathbf{p}, \mathbf{y}(t), \dot{\mathbf{y}}(t))
$$

où $\mathbf{u}(t)=\left(u_{1}(t), u_{2}(t)\right)^{T}, \mathbf{y}(t)=\left(y_{1}(t), y_{2}(t)\right)^{T}, \mathbf{p}=(a, b)^{T}$ et la fonction $\psi$ est définie par

$$
\psi=\left(\begin{array}{l}
\dot{y}_{1}(t)+b \sqrt{2 g y_{1}(t)}+a \cdot \operatorname{sign}\left(y_{1}(t)-y_{2}(t)\right) \sqrt{2 g\left|y_{1}(t)-y_{2}(t)\right|} \\
\dot{y}_{2}(t)+b \sqrt{2 g y_{2}(t)}-\operatorname{a.sign}\left(y_{1}(t)-y_{2}(t)\right) \sqrt{2 g\left|y_{1}(t)-y_{2}(t)\right|}
\end{array}\right) .
$$

Le système des bacs d'eau est donc un système inversible (de degré relatif 1 ).

Dans ce papier, nous nous intéressons au problème d'estimation des paramètres de systèmes inversibles non linéaires, décrient par (1), dans un contexte "en aveugle", c'est-à-dire où les entrées ne sont pas mesurées [AKH 03]. Seules les sorties y $(t)$ sont mesurées et des hypothèses statistiques faibles sur les entrées inconnus sont émises (comme l'indépendance).

Par exemple, considérons l'exemple 1 où seules les sorties $\left(y_{1}, y_{2}\right)$ sont mesurées. Si l'hypothèse d'indépendance des entrées $\left(u_{1}, u_{2}\right)$ est réaliste, les paramètres $(a, b)$ pourrait être estimés uniquement à partir de la connaissance des sorties, sans aucune autre mesure.

Notons que pour ce problème où l'information initiale est tellement pauvre, notre objectif est, dans un premier temps, de nous intéresser à l'identifiabilité des paramètres "en aveugle" (i.e. sans la connaissance des entrées). Nous poursuivrons ce papier en proposant une méthode d'estimation de $\mathbf{p}$ prouvant ainsi que la connaissance des entrées n'est pas toujours nécessaire à l'identification ; des hypothèses statistiques faibles peuvent suffire.

\subsection{Hypothèses sur les signaux}

Afin de résoudre ce problème d'estimation en aveugle, nous émettons des hypothèses statistiques $H_{i}$ sur le vecteur d'entrées $\mathbf{u}(t)$. L'ensemble de ces hypothèses $\mathbb{M}=\left\{H_{i}\right\}_{i}$ est appelé modèle (statistique) d'entrées pour $\mathbf{u}(t)$. Par la suite, on notera abusivement $\mathbf{u} \in \mathbb{M}$.

Étant donné un système inversible (1) et un modèle d'entrées $\mathbb{M}$, le problème d'estimation en aveugle consiste à caractériser l'ensemble des paramètres solutions suivant :

$$
\left\{\mathbf{p} \in \mathcal{P} \mid \psi\left(\mathbf{p}, \mathbf{y}(t), \ldots, \mathbf{y}^{(r)}(t)\right) \in \mathbb{M}\right\} .
$$

Dans ce papier, nous considérons uniquement le modèle d'entrées notés $\mathbb{M}_{\mathcal{I}_{s}}=$ $\left\{H_{1}, H_{2}\right\}$ où les hypothèses $H_{i}$ sont définies par :

$H_{1}=$ "Le vecteur d'entrées $\mathbf{u}$ appartient à $\mathcal{S}^{n}$, où $\mathcal{S}$ est l'ensemble des signaux aléatoires stationnaires, ergodiques et lisses". 
$H_{2}=$ "Le vecteur d'entrées u est à composantes indépendantes".

Remarque $2 S i \mathbf{u}$ satisfait $H_{1}$, alors $\dot{\mathbf{u}}, \ddot{\mathbf{u}}, \ldots, \mathbf{u}^{(k)}, \ldots$ appartiennent également $\grave{a}$ $\mathcal{S}^{n}$. Notons que cette hypothèse n'implique pas que le vecteur d'observations y appartienne à $\mathcal{S}^{n}$. Par exemple, si le système (1) est instable, y est non stationnaire et ne peut donc pas appartenir à $\mathcal{S}^{n}$.

\subsection{Fonctions d'estimation}

Une fonction d'estimation [CAR 97] est une fonction vectorielle de $\left(\mathbb{R}^{\mathbb{R}}\right)^{n}$ dans $\mathbb{R}^{q}$ dont les composantes font intervenir des moments statistiques. Par exemple, la fonction

$$
\mathbf{h}:\left\{\begin{array}{ccc}
\left(\mathbb{R}^{\mathbb{R}}\right)^{2} & \rightarrow & \mathbb{R} \\
\left(u_{1}, u_{2}\right) & \rightarrow & E\left(u_{1} u_{2}\right)-E\left(u_{1}\right) E\left(u_{2}\right)
\end{array}\right.
$$

est une fonction d'estimation. Ces fonctions sont construites de façon à s'annuler lorsque les hypothèses statistiques du modèle d'entrées $\mathbb{M}$ sont satisfaites. Par exemple, si les signaux d'entrées $u_{1}$ et $u_{2}$ sont supposés décorrélés, la fonction d'estimation (6) peut être utilisée.

Le problème d'estimation de paramètre en aveugle se formalise de la manière suivante : Soient $\mathbf{u}=\psi\left(\mathbf{p}, \mathbf{y}, \ldots, \mathbf{y}^{(r)}\right)$ un modèle paramétrique, où $\mathbf{y}$ est mesuré alors que $\mathbf{u}$ est inconnu, et $\mathbf{h}$ une fonction d'estimation pour le modèle statistique d'entrées $\mathbb{M}$, le problème d'estimation de paramètres en aveugle consiste à caractériser l'ensemble

$$
\mathbb{P}=\left\{\mathbf{p} \in \mathcal{P} \mid \mathbf{h}\left(\psi\left(\mathbf{p}, \mathbf{y}, \ldots, \mathbf{y}^{(r)}\right)\right)=\mathbf{0}\right\}
$$

\subsection{Fils conducteur}

Dans ce papier, nous présentons d'une part la notion d'identifiabilité structurelle en aveugle, extension de l'identifiabilité structurelle définit par Walter et Pronzato dans [WAL 97]. Cette nouvelle notion caractérise la possibilité d'estimer les paramètres $\mathbf{p}$ en exploitant uniquement les sorties et un modèle statistique $\mathbb{M}$ pour les entrées. Ainsi un système inversible sera qualifié d'identifiable en aveugle lorsque le modèle d'entrées $\mathbb{M}$ considéré est suffisamment riche pour retrouver le vecteur des paramètres de façon unique.

Ensuite, nous présentons une nouvelle méthodologie pour résoudre le problème d'estimation de paramètres en aveugle pour des systèmes inversibles, linéaires ou non. L'originalité de la méthodologie proposée repose sur l'utilisation conjointe de résultats peu connus (et par conséquent peu exploités) sur les dérivées de signaux aléatoires et de l'analyse par intervalle comme outils de résolutions [JAU 01].

Nous supposons qu'il n'existe aucun bruit de mesure. Ainsi, le vecteur des sorties 
$\mathbf{y}(t)$ et ses dérivées $\mathbf{y}^{(i)}(t)$ sont connus. Toutefois, même dans ce cadre idéal où $\mathbf{y}(t)$ et ses dérivées $\mathbf{y}^{(i)}(t)$ sont connues, il n'existe, à notre connaissance, aucune méthode générale pour estimer $\mathbf{p}$.

Remarque 3 Notons que l'identification de $\mathbf{p}$ implique la connaissance de $\mathbf{u}$ via la fonction $\psi$ et les signaux $\mathbf{y}^{(i)}$ connus (voir l'égalité (1)). Le problème d'estimation de paramètres en aveugle présente donc de fortes similitudes avec le problème de séparation aveugle de sources qui consiste à estimer les signaux d'entrées $\mathbf{u}$ à partir de l'observation de mélanges de ces signaux et à l'aide d'hypothèses statistiques sur ces derniers [JUT 91, COM 94, CIC 02, HYV 01, BEL 95, CAR 96].

Ce papier s'organise de la manière suivante. Dans la section 2, nous commençons par énoncer les principales propriétés de l'indépendance statistique qui seront exploitées dans la suite. Dans la section 3, nous formalisons le concept d'identifiabilité en aveugle et présentons des conditions d'identifiabilité en aveugle de systèmes inversibles paramétrés. La section 4 présente la méthodologie de résolution proposée dans ce papier. Cette méthode repose sur la construction de fonctions d'estimation adaptées au modèle d'entrées $\mathbb{M}$ considéré. Ces fonctions d'estimation, qui exploitent les résultats de la section 2 , ont pour rôle de traduire en terme d'équations les hypothèses statistiques du modèle d'entrées. Rappelons que nous nous intéresserons uniquement aux fonctions d'estimation pour le modèle d'entrées indépendant, noté $\mathbb{M}_{\mathcal{I}_{s}}$. Enfin, dans la section 5 , nous proposons une simulation illustrant notre démarche afin d'estimer en aveugle les paramètres du système inversible non linéaire de l'exemple 1.

\section{Quelques résultats concernant les signaux aléatoires indépendants}

Le problème d'estimation de paramètres en aveugle mettant en jeu des hypothèses statistiques sur les signaux d'entrées inconnues, nous commençons par introduire les propriétés essentielles du modèle d'entrées $\mathbb{M}_{\mathcal{I}_{s}}$ exploités dans ce papier. Ces propriétés seront utilisées afin, d'une part, d'établir des conditions d'identifiabilité en aveugle et, d'autre part, de construire des fonctions d'estimation adaptées en vue de l'identification des paramètres.

Cette partie rappelle quelques définitions et résultats concernant les dérivées de signaux aléatoires issues de [PIC 89, GUI 80, BLA 81]. Afin d'éviter d'allourdir les notations, les résultats présentés concernent uniquement des vecteurs de deux signaux alétaoires $\mathbf{u}=\left(u_{1}, u_{2}\right)^{T}$. La généralisation aux vecteurs de $n$ signaux aléatoires $\mathbf{u}=\left(u_{1}, \ldots, u_{n}\right)^{T}$ ne présente aucune difficulté. Commençons par énoncer la définition de l'indépendance de signaux aléatoires.

Définition 1 Soit $\mathcal{F}$ l'ensemble des fonctions définies de $\mathbb{R}^{\mathbb{R}}$ dans $\mathbb{R}$. Les signaux aléatoires $u_{1}$ et $u_{2}$ sont statistiquement indépendants si $\forall f_{1}, f_{2}, \in \mathcal{F}$, les variables aléatoires $x_{1}=f_{1}\left(u_{1}\right), x_{2}=f_{2}\left(u_{2}\right)$ sont statistiquement indépendantes, i.e.,

$$
p_{x_{1}, x_{2}}=p_{x_{1}} p_{x_{2}}
$$


avec $p_{x_{i}}$ et $p_{x_{1}, x_{2}}$, respectivement, les densités de probabilité marginales et conjointe des variables aléatoires $x_{i}$.

Dans la suite, l'indépendance de deux signaux aléatoires $u_{1}, u_{2}$ et deux variables aléatoires $x_{1}, x_{2}$ est respectivement noté $\mathcal{I}_{s}\left(u_{1}, u_{2}\right)$ et $\mathcal{I}_{v}\left(x_{1}, x_{2}\right)$.

Maintenant, la proposition suivante présente l'ensemble des fonctions qui conservent l'indépendance statistique.

Proposition 1 Soient $\mathfrak{F}$ l'ensemble des fonctions définies de $\mathbb{R}^{\mathbb{R}}$ à valeur dans $\mathbb{R}^{\mathbb{R}}$ et $\left(u_{1}, u_{2}\right)$ deux signaux aléatoires, on a

$$
\mathcal{I}_{s}\left(u_{1}, u_{2}\right) \Leftrightarrow \forall g_{1}, g_{2} \in \mathfrak{F}, \mathcal{I}_{s}\left(g_{1}\left(u_{1}\right), g_{2}\left(u_{2}\right)\right) .
$$

Preuve : $(\Rightarrow)$ Soient $g_{1}, g_{2} \in \mathfrak{F}$. D'après la définition 1 ,

$$
\mathcal{I}_{s}\left(g_{1}\left(u_{1}\right), g_{2}\left(u_{2}\right)\right) \Leftrightarrow \forall f_{1}, f_{2} \in \mathcal{F}, \mathcal{I}_{v}\left(f_{1}\left(g_{1}\left(u_{1}\right)\right), f_{2}\left(g_{2}\left(u_{2}\right)\right)\right) \text {. }
$$

Pour $f_{1}, f_{2} \in \mathcal{F}$, définissons les fonctions $h_{1}$ et $h_{2}$ telles que

$$
h_{i}:\left\{\begin{array}{ccc}
\mathbb{R}^{\mathbb{R}} & \rightarrow & \mathbb{R} \\
u_{i}(t) & \rightarrow & f_{i}\left(g_{i}\left(u_{i}(t)\right)\right)
\end{array} \quad, i=1,2 .\right.
$$

Sachant que $\mathcal{I}_{s}\left(u_{1}, u_{2}\right)$ et $h_{i} \in \mathcal{F}$, on a $\mathcal{I}_{v}\left(h_{1}\left(u_{1}\right), h_{2}\left(u_{2}\right)\right)$, i.e. $\mathcal{I}_{v}\left(f_{1}\left(g_{1}\left(u_{1}\right)\right), f_{2}\left(g_{2}\left(u_{2}\right)\right)\right)$. D'après (10), on obtient $\mathcal{I}_{s}\left(g_{1}\left(u_{1}\right), g_{2}\left(u_{2}\right)\right)$.

$(\Leftarrow)$ Trivial avec $g_{1}=g_{2}=I d$.

Exemple 2 Les deux fonctions suivantes

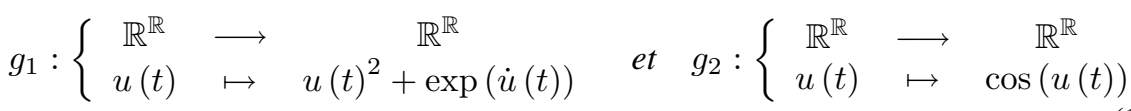

appartenant à $\mathfrak{F}$, on a $\mathcal{I}_{s}\left(u_{1}, u_{2}\right) \Rightarrow \mathcal{I}_{s}\left(u_{1}^{2}+\exp \left(\dot{u}_{1}\right), \cos \left(u_{2}\right)\right)$.

Remarque 4 Soient $u$ un signal aléatoire et $g_{1}, g_{2}$ deux fonctions de $\mathfrak{F}$, on a

$$
\left\{\begin{array}{l}
u_{1}=g_{1}(u) \\
u_{2}=g_{2}(u)
\end{array} \Rightarrow u_{1} \text { et } u_{2}\right. \text { sont dépendants. }
$$

\section{L'identifiabilité structurelle en aveugle}

Dans cette partie, nous définissons la notion d'identifiabilité (structurelle) en aveugle d'un système paramétré. Cette nouvelle notion, à la différence de l'identifiabilité structurelle [WAL 97, OLL 90], caractérise les chances de succés de l'estimation de paramètres d'un système sans en connaître ses entrées. En effet, avant même de résoudre de manière numérique le problème d'estimation en aveugle, se pose la question de l'unicité des paramètres solutions. En d'autres termes, les hypothèses $\mathbb{M}$ sur les entrées sont-elles suffisamment riches pour conduire à une estimation unique des paramètres? Dans cette partie, nous allons établir des conditions d'identifiabilité en aveugle pour le modèle particulier $\mathbb{M}_{\mathcal{I}_{s}}$ que nous illustrons sur des exemples simples. 


\subsection{Définition}

Considérons un système paramétré inversible de la forme $\mathbf{u}=\psi\left(\mathbf{p}, \mathbf{y}, \dot{\mathbf{y}}, \ldots, \mathbf{y}^{(r)}\right)$ et un modèle statistique $\mathbb{M}$ pour les entrées $\mathbf{u}($ i.e. $\mathbf{u} \in \mathbb{M})$.

Définition 2 Le système inversible $\mathbf{u}=\psi\left(\mathbf{p}, \mathbf{y}, \dot{\mathbf{y}}, \ldots, \mathbf{y}^{(r)}\right)$ est identifiable en aveugle selon le modèle d'entrée $\mathbb{M}$ si, $\forall \mathbf{p}^{\star} \in \mathcal{P}$, on a

$$
\left\{\begin{array}{l}
\psi\left(\mathbf{p}^{\star}, \mathbf{y}, \ldots, \mathbf{y}^{(r)}\right) \in \mathbb{M} \\
\psi\left(\hat{\mathbf{p}}, \mathbf{y}, \ldots, \mathbf{y}^{(r)}\right) \in \mathbb{M}
\end{array} \Longrightarrow \hat{\mathbf{p}}=\mathbf{p}^{\star},\right.
$$

En d'autres termes, pour un système est identifiable en aveugle, s'il suffit de trouver un vecteur de paramètres $\hat{\mathbf{p}}$ tel que $\hat{\mathbf{u}}=\psi\left(\hat{\mathbf{p}}, \mathbf{y}, \ldots, \mathbf{y}^{(r)}\right) \in \mathbb{M}$ pour que le vecteur $\hat{\mathbf{p}}$ ainsi obtenu soit une estimée de $\mathbf{p}^{\star}$. Notons que la notion d'identifiabilité en aveugle est définie relativement à un modèle d'entrées $\mathbb{M}$.

\subsection{L'identifiabilité en aveugle selon le modèle d'entrées indépendantes $\mathbb{M}_{\mathcal{I}_{s}}$}

Dans ce paragraphe, nous proposons des conditions d'identifiabilité en aveugle selon le modèle d'entrées $\mathbb{M}_{\mathcal{I}_{s}}$ de systèmes inversibles paramétrés. Nous allons voir que ces nouvelles conditions d'identifiabilité en aveugle permettent de tester algébriquement l'identifiabilité en aveugle d'un système inversible. Nous illustrerons ces résultats sur des exemples simples.

\subsubsection{Préliminaires}

Afin de simplifier la compréhension, nous allons introduire le système, noté $\mathcal{H}\left(\mathbf{p}^{\star}, \hat{\mathbf{p}}\right)$, qui associe à l'entrée $\left.\mathbf{u}^{\star}=\psi\left(\mathbf{p}^{\star}, \mathbf{y}, \ldots, \mathbf{y}^{(r)}\right)\right)$, l'entrée $\hat{\mathbf{u}}=\psi\left(\hat{\mathbf{p}}, \mathbf{y}, \ldots, \mathbf{y}^{(r)}\right)$ (voir figure 2). Plus formellement, pour un système inversible donné, le système $\mathcal{H}\left(\mathbf{p}^{\star}, \hat{\mathbf{p}}\right)$ est défini comme suit

$$
\begin{gathered}
\mathcal{H}\left(\mathbf{p}^{\star}, \hat{\mathbf{p}}\right):\left\{\begin{array}{ccc}
\mathbb{R}^{\mathbb{R}} & \rightarrow & \mathbb{R}^{\mathbb{R}} \\
\mathbf{u}^{\star} & \mapsto & \hat{\mathbf{u}}
\end{array}\right. \\
\operatorname{avec} \mathbf{u}^{\star}=\psi\left(\mathbf{p}^{\star}, \mathbf{y}, \ldots, \mathbf{y}^{(r)}\right) \text { et } \hat{\mathbf{u}}=\psi\left(\hat{\mathbf{p}}, \mathbf{y}, \ldots, \mathbf{y}^{(r)}\right) . \\
\mathcal{H}\left(\mathbf{p}^{\star}, \hat{\mathbf{p}}\right) \\
\mathbf{u}^{\star}=\psi\left(\mathbf{p}^{\star}, \mathbf{y}, \ldots, \mathbf{y}^{(r)}\right) \quad \mathbf{y}
\end{gathered}
$$

Figure 2. Système $\mathcal{H}\left(\mathbf{p}^{\star}, \hat{\mathbf{p}}\right)$.

Ce système s'obtient en deux étapes : Tout d'abord, il faut inverser le système $\mathbf{u}^{\star}=\psi\left(\mathbf{p}^{\star}, \mathbf{y}, \ldots, \mathbf{y}^{(r)}\right)$ afin d'obtenir une expression de la sortie $\mathbf{y}$. Cette étape est 
toujours possible, par hypothèse d'inversibilité. Ensuite, il convient d'injecter $\mathbf{y}$ dans le second système $\hat{\mathbf{u}}=\psi\left(\hat{\mathbf{p}}, \mathbf{y}, \ldots, \mathbf{y}^{(r)}\right)$ pour obtenir $\hat{\mathbf{u}}$.

L'exemple suivant illustre la construction du système $\mathcal{H}\left(\mathbf{p}^{\star}, \hat{\mathbf{p}}\right)$ d'un système inversible donné.

Exemple 3 Considèrons le système inversible des bacs d'eau de l'exemple 1 décrit par les équations (4). On a

$$
\left\{\begin{array}{l}
u_{1}^{\star}(t)=\dot{y}_{1}(t)+p_{2}^{\star} \sqrt{2 g y_{1}(t)}+p_{1}^{\star} \operatorname{sign}\left(y_{1}(t)-y_{2}(t)\right) \sqrt{2 g\left|y_{1}(t)-y_{2}(t)\right|} \\
u_{2}^{\star}(t)=\dot{y}_{2}(t)+p_{2}^{\star} \sqrt{2 g y_{2}(t)}-p_{1}^{\star} \operatorname{sign}\left(y_{1}(t)-y_{2}(t)\right) \sqrt{2 g\left|y_{1}(t)-y_{2}(t)\right|}
\end{array}\right.
$$

et

$$
\left\{\begin{array}{l}
\hat{u}_{1}=\dot{y}_{1}(t)+\hat{p}_{2} \sqrt{2 g y_{1}(t)}+\hat{p}_{1} \operatorname{sign}\left(y_{1}(t)-y_{2}(t)\right) \sqrt{2 g\left|y_{1}(t)-y_{2}(t)\right|} \\
\hat{u}_{2}=\dot{y}_{2}(t)+\hat{p}_{2} \sqrt{2 g y_{2}(t)}-\hat{p}_{1} \operatorname{sign}\left(y_{1}(t)-y_{2}(t)\right) \sqrt{2 g\left|y_{1}(t)-y_{2}(t)\right|}
\end{array}\right.
$$

L'expression du système $\mathcal{H}\left(\mathbf{p}^{\star}, \hat{\mathbf{p}}\right)$ est donc

$$
\left\{\begin{array}{l}
\dot{y}_{1}(t)=-p_{2}^{\star} \sqrt{2 g y_{1}(t)}-p_{1}^{\star} \operatorname{sign}\left(y_{1}(t)-y_{2}(t)\right) \sqrt{2 g\left|y_{1}(t)-y_{2}(t)\right|}+u_{1}^{\star}(t) \\
\dot{y}_{2}(t)=-p_{2}^{\star} \sqrt{2 g y_{2}(t)}+p_{1}^{\star} \operatorname{sign}\left(y_{1}(t)-y_{2}(t)\right) \sqrt{2 g\left|y_{1}(t)-y_{2}(t)\right|}+u_{2}^{\star}(t) \\
\hat{u}_{1}=u_{1}^{\star}(t)+\left(\hat{p}_{2}-p_{2}^{\star}\right) \sqrt{2 g y_{1}(t)}+\left(\hat{p}_{1}-p_{1}^{\star}\right) \operatorname{sign}\left(y_{1}(t)-y_{2}(t)\right) \sqrt{2 g\left|y_{1}(t)-y_{2}(t)\right|} \\
\hat{u}_{2}=u_{2}^{\star}(t)+\left(\hat{p}_{2}-p_{2}^{\star}\right) \sqrt{2 g y_{2}(t)}+\left(p_{1}^{\star}-\hat{p}_{1}\right) \operatorname{sign}\left(y_{1}(t)-y_{2}(t)\right) \sqrt{2 g\left|y_{1}(t)-y_{2}(t)\right|}
\end{array}\right.
$$

Le système $\mathcal{H}\left(\mathbf{p}^{\star}, \hat{\mathbf{p}}\right)$ s'écrit sous forme d'un modèle d'état où $\mathbf{y}$ est l'état, $\mathbf{u}^{\star}$ est l'entrée et $\hat{\mathbf{u}}$ est la sortie.

Le système $\mathcal{H}\left(\mathbf{p}^{\star}, \hat{\mathbf{p}}\right)$ peut être vue comme le "transfert" entre les entrées engendrées par les paramètres $\mathbf{p}^{\star}$ et celles engendrées par les paramètres $\hat{\mathbf{p}}$. L'introduction de ce système nous permet d'obtenir une condition nécessaire et suffisante d'identifiabilité en aveugle selon le modèle d'entrées indépendantes.

\subsubsection{Conditions d'identifiabilité en aveugle selon le modèle d'entrées indépendantes $\mathbb{M}_{\mathcal{I}_{s}}$}

Dans ce paragraphe, nous présentons une condition d'identifiabilité en aveugle selon le modèle d'entrées indépendantes.

Théorème $\mathbf{1}$ Le système $\mathbf{u}=\psi\left(\mathbf{p}, \mathbf{y}, \ldots, \mathbf{y}^{(r)}\right)$ est identifiable en aveugle selon le modèle d'entrées indépendantes si et seulement si, $\forall \mathbf{p}^{\star} \in \mathcal{P}$, on a l'implication suivante

$$
\mathcal{H}\left(\mathbf{p}^{\star}, \hat{\mathbf{p}}\right) \text { est découplé }^{1} \Longrightarrow \hat{\mathbf{p}}=\mathbf{p}^{\star}
$$

1. Un système est découplé si et seulement si chaque sortie ne dépend que d'une et une seule entrée et réciproquement. 
Preuve : Commençons par montrer l'équivalence suivante

$$
\mathcal{H}\left(\mathbf{p}^{\star}, \hat{\mathbf{p}}\right) \text { est découplé } \Leftrightarrow \mathcal{H}\left(\mathbf{p}^{\star}, \hat{\mathbf{p}}\right) \in \mathfrak{F} \text {. }
$$

Supposons que le système $\mathcal{H}\left(\mathbf{p}^{\star}, \hat{\mathbf{p}}\right)$ qui lie l'entrée $\mathbf{u}^{\star}$ et la sortie $\hat{\mathbf{u}}$ conserve l'indépendance, i.e. $\mathcal{H}\left(\mathbf{p}^{\star}, \hat{\mathbf{p}}\right) \in \mathfrak{F}$ (voir Proposition 1), on a

$$
\hat{u}_{i}=h_{i}\left(\mathbf{u}^{\star}\right) \text {, }
$$

avec $h_{i} \in \mathfrak{F}$. Or, d'après la contraposée de (12) (voir remarque 4), on en déduit que $\hat{u}_{i}=h_{i}\left(u_{j}^{\star}\right)$, c'est-à-dire que le système $\mathcal{H}\left(\mathbf{p}^{\star}, \hat{\mathbf{p}}\right)$ est découplé.

Si on suppose que le système $\mathcal{H}\left(\mathbf{p}^{\star}, \hat{\mathbf{p}}\right)$ est découplé, alors il conserve l'indépendance de façon trivial.

Par conséquent, l'équivalence (18) est vérifiée.

Maintenant, démontrons le théorème 1.

$(\Rightarrow)$ Supposons que le système $\mathbf{u}=\psi\left(\mathbf{p}, \mathbf{y}, \ldots, \mathbf{y}^{(r)}\right)$ est identifiable en aveugle selon le modèle d'entrées indépendantes, c'est-à-dire

$$
\left\{\begin{array}{l}
\mathbf{u}^{\star}=\psi\left(\mathbf{p}^{\star}, \mathbf{y}, \ldots, \mathbf{y}^{(r)}\right) \in \mathbb{M}_{\mathcal{I}_{s}} \\
\hat{\mathbf{u}}=\psi\left(\hat{\mathbf{p}}, \mathbf{y}, \ldots, \mathbf{y}^{(r)}\right) \in \mathbb{M}_{\mathcal{I}_{s}}
\end{array} \Rightarrow \hat{\mathbf{p}}=\mathbf{p}^{\star}\right.
$$

La contraposée cette l'implication nous donne

$$
\hat{\mathbf{p}} \neq \mathbf{p}^{\star} \Rightarrow\left\{\begin{array}{l}
\mathbf{u}^{\star}=\psi\left(\mathbf{p}^{\star}, \mathbf{y}, \ldots, \mathbf{y}^{(r)}\right) \notin \mathbb{M}_{\mathcal{I}_{s}} \\
\text { ou } \\
\hat{\mathbf{u}}=\psi\left(\hat{\mathbf{p}}, \mathbf{y}, \ldots, \mathbf{y}^{(r)}\right) \notin \mathbb{M}_{\mathcal{I}_{s}}
\end{array}\right.
$$

Or, par définition de $\mathfrak{F}$ et sachant que $\mathcal{H}\left(\mathbf{p}^{\star}, \hat{\mathbf{p}}\right)$ est le système qui lie les entrées $\mathbf{u}^{\star}$ et $\hat{\mathbf{u}}$, on a

$$
\left\{\begin{array}{l}
\mathbf{u}^{\star}=\psi\left(\mathbf{p}^{\star}, \mathbf{y}, \ldots, \mathbf{y}^{(r)}\right) \notin \mathbb{M}_{\mathcal{I}_{s}} \\
\text { ou } \\
\hat{\mathbf{u}}=\psi\left(\hat{\mathbf{p}}, \mathbf{y}, \ldots, \mathbf{y}^{(r)}\right) \notin \mathbb{M}_{\mathcal{I}_{s}}
\end{array} \Rightarrow \mathcal{H}\left(\mathbf{p}^{\star}, \hat{\mathbf{p}}\right) \notin \mathfrak{F} .\right.
$$

Par conséquent, en combinant (20) et (21), on obtient

$$
\hat{\mathbf{p}} \neq \mathbf{p}^{\star} \Rightarrow \mathcal{H}\left(\mathbf{p}^{\star}, \hat{\mathbf{p}}\right) \notin \mathfrak{F} .
$$

ou encore

$$
\mathcal{H}\left(\mathbf{p}^{\star}, \hat{\mathbf{p}}\right) \in \mathfrak{F} \Rightarrow \hat{\mathbf{p}}=\mathbf{p}^{\star}
$$

$(\Leftarrow)$ Supposons que

$$
\mathcal{H}\left(\mathbf{p}^{\star}, \hat{\mathbf{p}}\right) \in \mathfrak{F} \Rightarrow \hat{\mathbf{p}}=\mathbf{p}^{\star}
$$

Par définition de $\mathfrak{F}$, on a

$$
\left\{\begin{array}{l}
\mathbf{u}^{\star}=\psi\left(\mathbf{p}^{\star}, \mathbf{y}, \ldots, \mathbf{y}^{(r)}\right) \in \mathbb{M}_{\mathcal{I}_{s}} \\
\hat{\mathbf{u}}=\psi\left(\hat{\mathbf{p}}, \mathbf{y}, \ldots, \mathbf{y}^{(r)}\right) \in \mathbb{M}_{\mathcal{I}_{s}}
\end{array} \Rightarrow \mathcal{H}\left(\mathbf{p}^{\star}, \hat{\mathbf{p}}\right) \in \mathfrak{F}\right.
$$


10 Nom de la revue ou conférence (à définir par \submitted ou \toappear)

et d'après (24), on en déduit que

$$
\left\{\begin{array}{l}
\mathbf{u}^{\star}=\psi\left(\mathbf{p}^{\star}, \mathbf{y}, \ldots, \mathbf{y}^{(r)}\right) \in \mathbb{M}_{\mathcal{I}_{s}} \\
\hat{\mathbf{u}}=\psi\left(\hat{\mathbf{p}}, \mathbf{y}, \ldots, \mathbf{y}^{(r)}\right) \in \mathbb{M}_{\mathcal{I}_{s}}
\end{array} \Rightarrow \hat{\mathbf{p}}=\mathbf{p}^{\star}\right.
$$

c'est-à-dire que le système $\mathbf{u}=\psi\left(\mathbf{p}, \mathbf{y}, \ldots, \mathbf{y}^{(r)}\right)$ est identifiable en aveugle selon le modèle d'entrées indépendantes.

Nous illustrons cette condition d'identifiabilité en aveugle selon le modèle d'entrées indépendantes sur deux exemples simples.

Exemple 4 (Système non identifiable en aveugle) Considérons le système inversible

$$
\left(\begin{array}{l}
u_{1} \\
u_{2}
\end{array}\right)=\left(\begin{array}{cc}
1 & p_{1} \\
p_{2} & 1
\end{array}\right)\left(\begin{array}{l}
y_{1} \\
y_{2}
\end{array}\right)
$$

et le modèle d'entrées $\mathbb{M}_{\mathcal{I}_{s}}$ et déterminons le système $\mathcal{H}\left(\mathbf{p}^{\star}, \hat{\mathbf{p}}\right)$. Sachant que

$$
\left(\begin{array}{c}
u_{1}^{\star} \\
u_{2}^{\star}
\end{array}\right)=\left(\begin{array}{cc}
1 & p_{1}^{\star} \\
p_{2}^{\star} & 1
\end{array}\right)\left(\begin{array}{l}
y_{1} \\
y_{2}
\end{array}\right) \Rightarrow\left(\begin{array}{l}
y_{1} \\
y_{2}
\end{array}\right)=\frac{1}{1-p_{1}^{\star} p_{2}^{\star}}\left(\begin{array}{cc}
1 & -p_{1}^{\star} \\
-p_{2}^{\star} & 1
\end{array}\right)\left(\begin{array}{l}
u_{1}^{\star} \\
u_{2}^{\star}
\end{array}\right),
$$

le système $\mathcal{H}\left(\mathbf{p}^{\star}, \hat{\mathbf{p}}\right)$ s'écrit

$$
\begin{aligned}
\left(\begin{array}{c}
\hat{u}_{1} \\
\hat{u}_{2}
\end{array}\right) & =\left(\begin{array}{cc}
1 & \hat{p}_{1} \\
\hat{p}_{2} & 1
\end{array}\right) \underbrace{\frac{1}{1-p_{1}^{\star} p_{2}^{\star}}\left(\begin{array}{cc}
1 & -p_{1}^{\star} \\
-p_{2}^{\star} & 1
\end{array}\right)\left(\begin{array}{c}
u_{1}^{\star} \\
u_{2}^{\star}
\end{array}\right)}_{\mathbf{y}} \\
& =\frac{1}{1-p_{1}^{\star} p_{2}^{\star}}\left(\begin{array}{cc}
1-\hat{p}_{1} p_{2}^{\star} & -p_{1}^{\star}+\hat{p}_{1} \\
-p_{2}^{\star}+\hat{p}_{2} & 1-p_{1}^{\star} \hat{p}_{2}
\end{array}\right)\left(\begin{array}{c}
u_{1}^{\star} \\
u_{2}^{\star}
\end{array}\right) .
\end{aligned}
$$

La condition (1) devient, $\forall \mathbf{p}^{\star} \in \mathcal{P}$,

$$
(\left(\begin{array}{c}
\hat{u}_{1} \\
\hat{u}_{2}
\end{array}\right)=\frac{1}{1-p_{1}^{\star} p_{2}^{\star}} \underbrace{\left(\begin{array}{cc}
1-\hat{p}_{1} p_{2}^{\star} & -p_{1}^{\star}+\hat{p}_{1} \\
-p_{2}^{\star}+\hat{p}_{2} & 1-p_{1}^{\star} \hat{p}_{2}
\end{array}\right)}_{\mathbf{M}}\left(\begin{array}{c}
u_{1}^{\star} \\
u_{2}^{\star}
\end{array}\right) \text { est découplé } \Longrightarrow \hat{\mathbf{p}}=\mathbf{p}^{\star} .)
$$

Or, $\mathcal{H}\left(\mathbf{p}^{\star}, \hat{\mathbf{p}}\right)$ est découplé si et seulement si la matrice $\mathbf{M}$ est diagonal ou antidiagonale, c'est-à-dire si et seulement si

$$
\left\{\begin{array} { l } 
{ 1 - \hat { p } _ { 1 } p _ { 2 } ^ { \star } = 0 } \\
{ 1 - p _ { 1 } ^ { \star } \hat { p } _ { 2 } = 0 }
\end{array} \text { ou } \left\{\begin{array}{l}
-p_{1}^{\star}+\hat{p}_{1}=0 \\
-p_{2}^{\star}+\hat{p}_{2}=0
\end{array}\right.\right. \text {. }
$$

Et, en résolvant ces deux systèmes d'équations d'inconnus $\hat{\mathbf{p}}$, on obtient

$$
\left\{\begin{array} { l } 
{ \hat { p } _ { 1 } = \frac { 1 } { p _ { 2 } ^ { \star } } } \\
{ \hat { p } _ { 2 } = \frac { 1 } { p _ { 1 } ^ { \star } } }
\end{array} \text { et } \left\{\begin{array}{l}
\hat{p}_{1}=p_{1}^{\star} \\
\hat{p}_{2}=p_{2}^{\star}
\end{array}\right.\right. \text {. }
$$

Donc, d'après le théorème 1, le système (27) n'est pas identifiable en aveugle selon $\mathrm{M}_{\mathcal{I}_{s}}$ (voir solutions du système d'équations (28)). 
Exemple 5 (Système identifiable en aveugle) Considérons le système des bacs d'eau de l'exemple 1 décrit par les équations (2). Le système $\mathcal{H}\left(\mathbf{p}^{\star}, \hat{\mathbf{p}}\right)$ défini par (17) est découplé si chaque sortie est fonction d'une seule entrée, c'est-à-dire si $u_{1}^{\star}=g_{1}\left(\hat{u}_{1}\right)$ et $u_{2}^{\star}=g_{2}\left(\hat{u}_{2}\right)^{2}$. Pour assurer cette condition de découplage, $\hat{u}_{1}$ ne doit dépendre $n i$ de $y_{2}$ (puisque $\dot{y}_{2}$ fait intervenir $u_{2}^{\star}$ ) et ni de $y_{1}$ (puisque $\dot{y}_{1}$ fait intervenir $\dot{y}_{2}$ ). Et, de la même façon, $\hat{u}_{2}$ ne doit pas dépendre de $y_{1}$ et $y_{2}$. On doit donc avoir

$$
\left\{\begin{array}{l}
\hat{p}_{2}-p_{2}^{\star}=0 \\
\hat{p}_{1}-p_{1}^{\star}=0
\end{array} .\right.
$$

Et donc, il faut nécessairement $\hat{p}_{1}=p_{1}^{\star}$ et $\hat{p}_{2}=p_{2}^{\star}$ (ainsi $\hat{\mathbf{u}}=\mathbf{u}^{\star}$ ). Donc, d'après le théorème 1 , le système (2) est identifiable en aveugle selon le modèle d'entrée indépendantes $\mathbb{M}_{\mathcal{I}_{s}}$.

\section{Méthode d'estimation en aveugle}

Rappelons que la résolution du problème d'estimation de paramètres en aveugle repose sur la construction de fonction d'estimation adaptée dont le rôle est de traduire les hypothèses statistiques $\mathbb{M}$ sur les signaux d'entrées en équations (appelées équations d'estimation) du type $\mathbf{h}(\mathbf{u})=\mathbf{0}$ (voir paragraphe 1.2). Ainsi, pour chaque modèle d'entrées $\mathbb{M}$, nous allons construire une fonction d'estimation $\mathbf{h}$ adaptée de telle sorte que

$$
\mathbf{u} \in \mathbb{M} \Longrightarrow \mathbf{h}(\mathbf{u})=\mathbf{0} \text {. }
$$

et de caractériser l'ensemble $\mathbb{P}=\left\{\mathbf{p} \in \mathcal{P} \mid \mathbf{h}\left(\psi\left(\mathbf{p}, \mathbf{y}, \ldots, \mathbf{y}^{(r)}\right)\right)\right\}$.

Remarque 5 Notons que, bien que la fonction d'estimation $\mathbf{h}$ soit nulle, il est possible que les signaux $\mathbf{u}$ ne vérifient pas $\mathbb{M}$. Trouver une fonction h de telle sorte que la condition de suffisance pour 30 soit vérifiée est un problème extrêmement complexe au delà de l'objectif de ce papier. Cependant, dans la mesure où les techniques d'analyse par intervalles mises en oeuvre sont garanties (aucun solution n'est perdu)[JAU 01], l'ensemble des zeros de $\mathbf{h}$ est déterminé. Ainsi, si plusieurs zeros pour $\mathbf{h}$ sont obtenus alors que le système considéré est identifiable en aveugle (voir paragraphe 3.2.2 pour tester l'identifiabilité en aveugle), le nombre de composantes pour $\mathbf{h}$ peut être augmenté afin d'identifier les paramètres solutions de façon unique. Inversement, considérons maintenant un système complexe pour lequel il est impossible de tester l'identifiabilité en aveugle de ces paramètres. Si l'ensemble des zeros de $\mathbf{h}$ est un singleton, on peut garantir (a posteriori) l'identifiabilité en aveugle des paramètres.

Dans un premier temps, nous montrerons comment de telles fonctions peuvent être construites dans le cas particulier du modèle d'entrées $\mathbb{M}_{\mathcal{I}_{s}}$ considéré jusqu'à présent. Puis, pour terminer, nous approximons ces fonctions afin d'obtenir une expression analytique caractérisant l'ensemble solution $\mathbb{P}$.

2. La condition (de découplage) équivalente $u_{2}^{\star}=g_{1}\left(\hat{u}_{1}\right)$ et $u_{1}^{\star}=g_{2}\left(\hat{u}_{2}\right)$ est ici impossible, puisque $u_{1}^{\star}$ dépendent explicitement de $\hat{u}_{1}$ et $u_{2}^{\star}$ de $\hat{u}_{2}$. 
12 Nom de la revue ou conférence (à définir par \submitted ou \toappear)

\subsection{Fonction d'estimation pour le modèle d'entrées indépendantes $\mathbb{M}_{\mathcal{I}_{s}}$}

Commençons par énonçons quelques propriétés, utilisées par la suite, concernant les moments d'un vecteur de signaux aléatoires stationnaires, ergodiques et lisses $\mathbf{u}=\left(u_{1}, u_{2}\right)^{T} \in \mathcal{S}^{2}$.

Proposition 2 La fonction d'intercorrélation des dérivées d'un vecteur de signaux aléatoires $\mathbf{u}=\left(u_{1}, u_{2}\right)^{T} \in \mathcal{S}^{2}$ définie par

$$
\Gamma_{u_{i}^{(k)}, u_{j}^{(\ell)}}(\tau)=E\left(u_{i}^{(k)}(t) u_{j}^{(\ell)}(t-\tau)\right)-E\left(u_{i}^{(k)}(t)\right) E\left(u_{j}^{(\ell)}(t-\tau)\right),
$$

avec $k \geq 0, \ell \geq 0, i, j \in\{1,2\}$, vérifie

$$
\begin{aligned}
& \text { (i) } \Gamma_{u_{i}^{(k)}, u_{j}^{(\ell)}}(\tau)=E\left(u_{i}^{(k)}(t) u_{j}^{(\ell)}(t-\tau)\right) \text { si } k+\ell \geq 1, \\
& \text { (ii) } k_{1}+\ell_{1}=k_{2}+\ell_{2} \Rightarrow \Gamma_{u_{i}^{\left(k_{1}\right)}, u_{j}^{\left(\ell_{1}\right)}}(\tau)=(-1)^{\ell_{1}-\ell_{2}} \Gamma_{u_{i}^{\left(k_{2}\right)}, u_{j}^{\left(\ell_{2}\right)}}(\tau) .
\end{aligned}
$$

Preuve : Voir [BLA 81]. L'idée de la preuve repose sur les deux égalités suivantes :

$$
E\left(u_{i}^{(k)}\right)=0, \forall k \geq 1 \text { et } \Gamma_{u_{i}^{(k)} u_{j}^{(\ell)}}(\tau)=(-1)^{\ell} \frac{d^{k+\ell} \boldsymbol{\Gamma}_{u_{i} u_{j}}(\tau)}{d^{k+\ell} \tau} .
$$

Supposons que le modèle pour les signaux d'entrées $\mathbf{u}$ est $\mathbb{M}_{\mathcal{I}_{s}}$, i.e. $\mathcal{I}_{s}\left(u_{1}, \ldots, u_{n}\right)$. La dérivation conservant l'indépendance, on a également $\mathcal{I}_{s}\left(u_{1}^{\left(k_{1}\right)}, \ldots, u_{n}^{\left(k_{n}\right)}\right), \forall k_{i} \in$ $\mathbb{N}$ (voir proposition 1). Donc, pour tout entier $k, l \geq 0$, on a

$$
\Gamma_{u_{i}^{(k)}, u_{j}^{(l)}}(\tau)=0 . \quad\left(u_{i}^{(k)} \text { et } u_{j}^{(l)} \text { sont décorrélés }\right)
$$

Pour simplifier, nous posons $\tau=0$ afin d'utiliser uniquement l'influence des dérivations successives des fonctions d'intercorrélation et notons $\Gamma_{u_{i}^{(k)}, u_{j}^{(l)}} \equiv \Gamma_{u_{i}^{(k)}, u_{j}^{(l)}}(0)$. D'autre part, en remarquant d'après la proposition 2 qu'il existe des relations entre certains moments (par exemple $\Gamma_{u_{i}^{(2)}, u_{j}}=-\Gamma_{u_{i}^{(1)}, u_{j}^{(1)}}$ ) et dans le but d'éliminer ces termes redondants, nous choisissons la fonction d'estimation suivante :

$$
\mathbf{h}:\left\{\begin{aligned}
\left(\mathbb{R}^{\mathbb{R}}\right)^{n} & \rightarrow \mathbb{R}^{(q+1) \times n} \\
\mathbf{u} & \mapsto\left(\Gamma_{u_{i}^{(k)}, u_{j}}\right)_{i=1, \ldots, n-1, j=i+1, \ldots, n}^{k=0, \ldots, q}
\end{aligned}\right.
$$

où l'entier $q$ est l'ordre maximal de dérivation des signaux d'entrées.

Exemple 6 Pour deux signaux d'entrées $u_{1}$ et $u_{2}$, la fonction $\mathbf{h}$ définie par

$$
\mathbf{h}(\mathbf{u})=\left(\Gamma_{u_{1}, u_{2}}, \Gamma_{u_{1}^{(1)}, u_{2}}, \Gamma_{u_{1}^{(2)}, u_{2}}\right)^{T}
$$

est une fonction d'estimation pour le modèle d'entrées indépendantes $\mathbb{M}_{\mathcal{I}_{s}}$. 
En pratique, l'entier $q$ est choisi de façon à ce que le nombre d'équations d'estimation (les composantes de $\mathbf{h}$ ) soit supérieur ou égal au nombre d'inconnues (les paramètres). Plus $q$ est grand, plus le nombre d'équations est grand par rapport au nombre d'inconnues, et on peut ainsi espérer obtenir une estimation robuste.

Remarquons que nous utilisons uniquement des moments d'ordre deux dans la fonction d'estimation (35). Toutefois, des moments d'ordres supérieurs (comme par exemple $\left.E\left(\dot{u}_{i}^{3} u_{j}\right), E\left(\dot{u}_{i} \ddot{u}_{j}^{5}\right), \ldots\right)$ peuvent être ajoutés [J.L 97].

\subsection{Approximation des fonctions d'estimations}

Rappelons que l'ensemble $\mathbb{P}$ est défini par l'équation vectorielle suivante (voir l'équation (7)) :

$$
\mathbf{g}(\mathbf{p}) \triangleq \mathbf{h}\left(\psi\left(\mathbf{p}, \mathbf{y}, \dot{\mathbf{y}}, \ldots, \mathbf{y}^{(r)}\right)\right)=\mathbf{0}
$$

Dans la pratique, il est impossible d'obtenir une expression analytique de la fonction g. En effet, si le système $\psi$ est non linéaire ou instable, alors le signal y est rarement stationnaire, même si $\mathbf{u} \in \mathcal{S}^{n}$. Et, même si les signaux sont stationnaires, les espérances statistiques ne peuvent qu'être estimés. Nous allons détailler, sur un exemple simple, comment il est possible d'obtenir une estimation empirique de $\mathrm{g}$.

Considérons le système inversible décrit par l'équation suivante

$$
u=\psi(p, y, \dot{y})=\dot{y}+p \sin y,
$$

et supposons que la fonction d'estimation choisi est $h(u)=E(u \dot{u})$. Sachant que $u$ est stationnaire (par hypothèse), on a

$$
\begin{aligned}
g(p) & =h(\dot{y}+p \sin y), \\
& =E\left(\dot{y} \ddot{y}+p \dot{y}^{2} \cos y+p \ddot{y} \sin y+p^{2} \dot{y} \sin y \cos y\right) .
\end{aligned}
$$

Par contre, les signaux $\dot{y} \ddot{y}, \dot{y}^{2} \cos y, \ldots$ sont non stationnaires et il n'est pas possible de distribuer l'opérateur espérance.

Définissons l'opérateur bar d'un signal (stationnaire ou non) par

$$
\bar{x} \stackrel{\text { def }}{=} \frac{1}{N} \sum_{k=0}^{N} x(k \tau),
$$

avec $N$ le nombre d'échantillons disponibles et $\tau$ la période d'échantillonnage. Cette opérateur linéaire est un estimateur empirique de l'espérance $E(x)$.Donc $g(p)$ peut être approchée par

$$
\begin{aligned}
\hat{g}(p) & =\overline{\dot{y} \ddot{y}+p \dot{y}^{2} \cos y+p \ddot{y} \sin y+p^{2} \dot{y} \sin y \cos y} \\
& =\overline{\dot{y} \ddot{y}}+p \overline{\dot{y}^{2} \cos y}+p \bar{y} \sin y+p^{2} \overline{\dot{y} \sin y \cos y} .
\end{aligned}
$$

La fonction $g(p)$ est approximée par un polynôme du second degré $\hat{g}(p)$ dont les coefficients sont connus puisque le signal $y$ est connu. 
14 Nom de la revue ou conférence (à définir par \submitted ou \toappear)

\section{Simulation}

Dans cette partie, nous considérons le système des bacs d'eau de l'exemple 1. Nous supposons que les entrées inconnues $\mathbf{u}$ sont indépendantes (modèle d'entrées $\mathbb{M}_{\mathcal{I}_{s}}$ ), puis nous construisons une fonction d'estimation adaptée afin d'estimer en aveugle les paramètres inconnus $\mathbf{p}=(a, b)$ à partir de la mesure des sorties $\mathbf{y}$.

Rappelons que le système considéré est décrit par les équations suivantes :

$$
\left(\begin{array}{l}
u_{1} \\
u_{2}
\end{array}\right)=\left(\begin{array}{l}
\dot{y}_{1}(t)+b \sqrt{2 g y_{1}(t)}+\operatorname{assign}\left(y_{1}(t)-y_{2}(t)\right) \sqrt{2 g\left|y_{1}(t)-y_{2}(t)\right|} \\
\dot{y}_{2}(t)+b \sqrt{2 g y_{2}(t)}-\operatorname{arsign}\left(y_{1}(t)-y_{2}(t)\right) \sqrt{2 g\left|y_{1}(t)-y_{2}(t)\right|}
\end{array}\right) .
$$

Pour la simulation de ce système, nous considérons deux entrées $u_{1}$ et $u_{2}$ indépendantes $\mathcal{I}_{s}\left(u_{1}, u_{2}\right)$ et gaussiennes $\mathcal{G}_{s}\left(u_{1}\right), \mathcal{G}_{s}\left(u_{2}\right)$ obtenues par filtrage d'un bruit blanc gaussien de $N=9000$ échantillons avec une période de 0.01 (voir figure 3 ). A noter que par la suite, le caractère gaussien des entrées n'est pas exploitée. Les deux valeurs des paramètres sont $p_{1}^{\star}=a^{\star}=0.3$ et $p_{2}^{\star}=b^{\star}=0.5$. Les sorties correspondantes sont représentées sur la figure 4 .
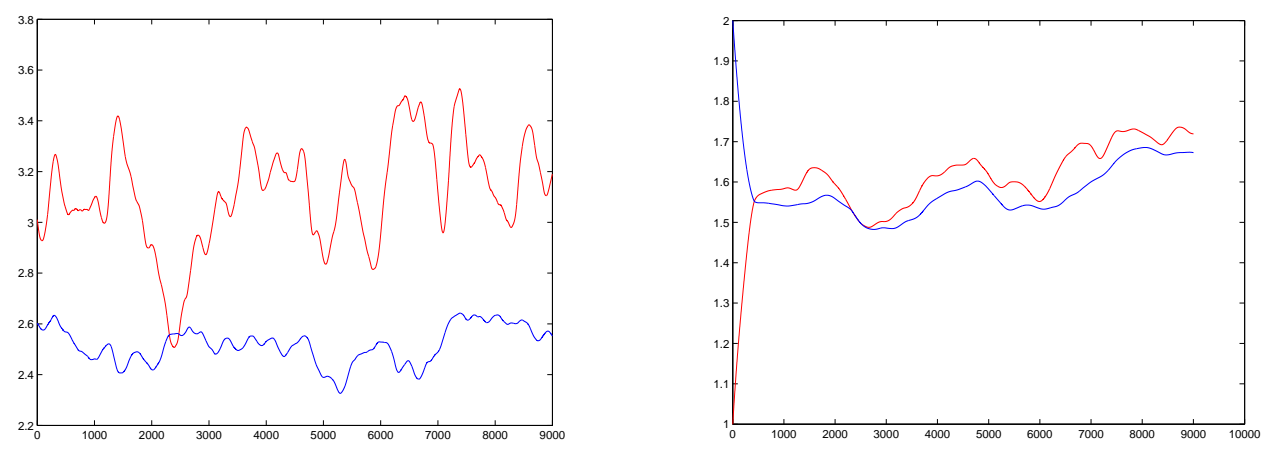

Figure 3. Entrées $u_{1}(t)$ (en clair) et $u_{2}(t)$ (en Figure 4. Sorties $y_{1}(t)$ (en clair) et $y_{2}(t)$ (en foncé). foncé).

Maintenant, estimons en aveugle les paramètres du système (41). Les paramètres $\mathbf{p}$ et les entrées $\mathbf{u}$ sont considérés inconnus ; seules les sorties sont connues. Nous supposons uniquement que les entrées sont indépendantes, $\mathcal{I}_{s}\left(u_{1}, u_{2}\right)$. Avant de construire une fonction d'estimation pour ce modèle d'entrées qui nous permettra d'estimer les paramètres, rappelons que ce système est identifiable en aveugle selon $\mathbb{M}_{\mathcal{I}_{s}}$ comme cela a été montré à l'occasion de l'exemple 5.

Maintenant, d'après les résultats du paragraphe 4.1, nous choisissons la fonction d'estimation $\mathbf{h}$ suivante :

$$
\mathbf{h}=\left(\begin{array}{c}
\Gamma_{u_{1} u_{2}} \\
\Gamma_{\dot{u}_{1} u_{2}}
\end{array}\right)=\left(\begin{array}{c}
E\left(u_{1} u_{2}\right)-E\left(u_{1}\right) E\left(u_{2}\right) \\
E\left(\dot{u}_{1} u_{2}\right)
\end{array}\right)
$$

qui founit deux équations (autant que de paramètres inconnus). Cette fonction d'estimation s'annule lorsque les entrées $u_{1}$ et $u_{2}$, et $\dot{u}_{1}$ et $u_{2}$ sont décorélées. A noter 
que nous nous plaçons ici dans la situation limite où l'on dispose d'autant d'équations que d'inconnus, toutefois, il est clair que nous pouvons ajouter des composantes supplémentaires à la fonction $\mathbf{h}$ afin d'améliorer l'estimation et ainsi exploiter plus en profondeur l'hypothèse d'indépendance.

Par une démarche similaire au paragraphe 4.2 , la condition $\mathbf{h}(\mathbf{u})=\mathbf{0}$ peut être approximée par

$$
\left\{\begin{array}{ccc}
\overline{u_{1} u_{2}}-\overline{u_{1}} \overline{u_{2}} & =0 \\
\overline{u_{1} u_{2}} & =0
\end{array}\right.
$$

D'après les équations (41) et sachant que

$$
\dot{u}_{1}(t)=\ddot{y}_{1}(t)+\frac{\sqrt{g} b \dot{y}_{1}(t)}{\sqrt{2 y_{1}(t)}}+\frac{\sqrt{g} a \dot{y}_{1}(t)}{\sqrt{2\left|y_{1}(t)-y_{2}(t)\right|}},
$$

on obtient

$$
\begin{aligned}
& \overline{u_{1}} \quad=\overline{\dot{y}_{1}}+b \sqrt{2 g} \overline{\sqrt{y_{1}}}+a \sqrt{2 g} \overline{\operatorname{sign}\left(y_{1}-y_{2}\right) \sqrt{\left|y_{1}-y_{2}\right|}}, \\
& \overline{u_{2}} \quad=\overline{y_{2}}+b \sqrt{2 g} \overline{\sqrt{y_{2}}}-a \sqrt{2 g} \overline{\operatorname{sign}\left(y_{1}-y_{2}\right) \sqrt{\left|y_{1}-y_{2}\right|}}, \\
& \begin{array}{l}
\overline{\dot{y}_{1} \dot{y}_{2}}+b \sqrt{2 g} \overline{\dot{y}_{2} \sqrt{y_{1}}}+a \sqrt{2 g} \overline{\dot{y}_{2} \operatorname{sign}\left(y_{1}-y_{2}\right) \sqrt{\left|y_{1}-y_{2}\right|}} \\
\overline{u_{1} u_{2}}=\quad+\sqrt{2 g} b \overline{\dot{y}_{1 \sqrt{y_{2}}}}+2 g b^{2} \overline{\sqrt{y_{1} y_{2}}}+2 g a b \operatorname{sign}\left(y_{1}-y_{2}\right) \sqrt{y_{2}\left|y_{1}-y_{2}\right|}
\end{array} \\
& -a \sqrt{2 g} \overline{\dot{y}_{1} \operatorname{sign}\left(y_{1}-y_{2}\right) \sqrt{\left|y_{1}-y_{2}\right|}} \\
& -a b \sqrt{2 g} \overline{\sqrt{2 g y_{1}} \operatorname{sign}\left(y_{1}-y_{2}\right) \sqrt{2 g\left|y_{1}-y_{2}\right|}}-2 a^{2} g \overline{\left|y_{1}-y_{2}\right|}
\end{aligned}
$$

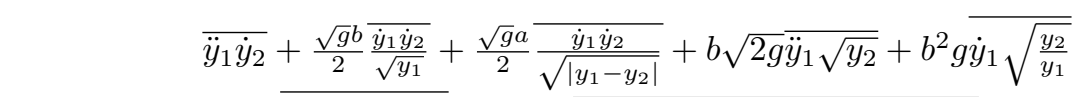

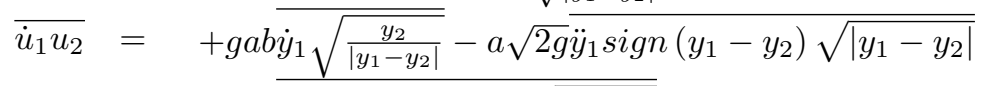

$$
\begin{aligned}
& -a g b \dot{y}_{1} \operatorname{sign}\left(y_{1}-y_{2}\right) \sqrt{\frac{\left|y_{1}-y_{2}\right|}{y_{1}}}-g a^{2} \overline{\dot{y}_{1} \operatorname{sign}\left(y_{1}-y_{2}\right)} \text {. }
\end{aligned}
$$

Le système d'équations (43) est composé de deux équations non linéaires d'inconnues $a$ et $b$ dont les coefficients $\left(\overline{\dot{y}_{1} \dot{y}_{2}}, \overline{\sqrt{y_{1}}}, \overline{\dot{y}_{2}}, \ldots\right)$ dépendent de $N$. Nous avons résolu ce système pour différentes valeurs de $N$ en utilisant une méthode d'analyse par intervalles (voir [JAU 01], [MOO 79]). Pour chaque $N$, nous trouvons un unique vecteur solution $\hat{\mathbf{p}}(N)$. Le tableau (5) représente l'erreur quadratique moyenne d'estimation pour différentes valeur de $N$. On peut vérifier que $\hat{\mathbf{p}}(N)$ tend vers $\mathbf{p}^{*}$, la valeur exacte des paramètres, lorsque $N$ augmente.

\section{Conclusion}

Dans ce papier, nous nous sommes intéressés au problème d'estimation des paramètres d'un système lorsque ses entrées sont inconnues. Précisons que, dans ce 
16 Nom de la revue ou conférence (à définir par \submitted ou \toappear)

\begin{tabular}{|c|c|c|c|c|c|c|}
\hline$N$ & 100 & 600 & 1000 & 6000 & 10000 & 16000 \\
\hline$\left(\hat{p}_{1}(N)-p_{1}^{*}\right)^{2}$ & $4,8.10^{-2}$ & $1,1.10^{-2}$ & $1,1.10^{-2}$ & $10^{-4}$ & $2,5.10^{-5}$ & $10^{-5}$ \\
\hline$\left(\hat{p}_{2}(N)-p_{2}^{*}\right)^{2}$ & $7,8.10^{-2}$ & $3,5.10^{-2}$ & $1,4.10^{-2}$ & $10^{-3}$ & $9.10^{-5}$ & $3.10^{-6}$ \\
\hline
\end{tabular}

Tableau 1. Erreur quadratique moyenne d'estimation en aveugle des paramètres à partir de l'hypothèse d'indépendance.

contexte difficile, notre objectif n'était pas de fournir une estimation robuste des paramètres, mais simplement de montrer que ce problème, basiquement considéré insoluble, peut être résolu en utilisant des hypothèses faibles sur les entrées. L'interêt de ces hypothèses faibles est qu'elles s'appliquent pour une grande gamme de signaux et donc de problèmes.

Dans un premier temps, après avoir présenté quelques résultats généraux sur les dérivées de signaux aléatoires, nous avons introduit la notion d'identifiabilité en aveugle selon un modèle d'entrée. Cette nouvelle notion, fortement inspirée de l'identifiabilité structurelle, caractérise les chances de succès de l'identification des paramètres sans connaître les entrées. Nous avons, en particulier, étudié le deux modèle d'entrées indépendantes, pour lequel nous avons établi des conditions formelles d'identifiabilité en aveugle.

Dans un second temps, nous avons proposé une démarche originale de résolution du problème d'estimation en aveugle pour une grande classe de système paramétré (incluant les systèmes plats). Notre méthode de résolution repose sur la construction de fonctions d'estimation (exploitant les résultats sur les dérivées de signaux aléatoires) qui permettent d'estimer les paramètres. Pour ce problème, l'information initiale est tellement pauvre qu'il nous a paru intéressant d'aller chercher des renseignements supplémentaires dans les dérivées des signaux de sorties. Et, même dans le cadre idéal où les dérivées des signaux de sorties sont connues, il n'existait, à notre connaissance, aucune méthode permettant de résoudre ce problème d'estimation sans la connaissance des entrées.

Ainsi, bien que les entrées soient inconnues, nous avons montré qu'uniquement à partir d'hypothèses statistiques sur ces dernières, l'estimation des paramètres était possible. Malheureusement, nous ne sommes pas encore capables de présenter des conditions rigoureuses de succès de notre démarche dans la mesure où il existe sans doute des distributions de signaux d'entrées pathologiques pour lesquelles les fonctions d'estimation choisies se révèlent inadaptées. Par exemple, les composantes de la fonction d'estimation peuvent être linéairement dépendantes faisant ainsi échouer notre méthode.

Toutefois, la méthode de résolution que nous proposons utilise des techniques d'analyse par intervalles qui ont l'avantage être globales et garanties. Ainsi, bien qu' aucune preuve d'unicité des paramètres n'est donnée, notre méthode fournit l'ensemble des paramètres compatibles (avec la fonction d'estimation choisie). L'intérêt de l'utilisation des outils d'analyse par intervalles est majeur puisque, même dans le cas où l'identifiablilité en aveugle d'un système ne peut être testée (par exemples pour des 
systèmes complexes), l'analyse par intervalles nous donne une information a posteriori sur l'identifiabilité en aveugle du système. En effet, si une unique solution est obtenue (comme ces le cas dans de l'exemple présenté), alors le système considéré est, sans aucun doute, identifiable en aveugle selon le modèle fixé.

Pour conclure, précisons que notre méthode de résolution (qui exploite autant d'équations que d'inconnues) n'est clairement pas robuste. En effet, lorsque les sorties sont bruitées, lorsque le nombre d'échantillons est faible, lorsque le modèle n'est pas parfaitement connu (e.g. l'ordre $r$ est inconnu) ou lorsque les signaux d'entrées sont non stationnaires, la méthode proposée ne conduit pas a une estimation efficace des paramètres. En perspectives, il serait donc intéressant de réaliser une étude de robustesse en particulier vérifier que lorsque les sorties sont bruitées ou lorsque peu d'échantillons sont disponibles, l'estimation des paramètres est encore possible moyennant l'ajout d'équations supplémentaires.

\section{Bibliographie}

[AKH 03] AKhenak A., Cahldi M., Maquin D., Ragot J., « Sliding mode multiple observer for fault detection and isolation », 42nd IEEE Conf. on Decision and Control, vol. 1, Hawaii, USA, december 2003, p. 953-958.

[BEL 95] Bell T., SejNows KI T., « An Information-Maximization Approach to Blind Separation and Blind Deconvolution », Neural Computation, vol. 7, 1995, p. 1004-1034.

[BLA 81] Blanc-Lapierre A., Picinbono B., Fonctions Aléatoires, Masson, Paris, 1981.

[CAR 96] CARdoso J. F., LAheld B., « Equivariant Adaptive Source Separation. », IEEE Transactions on Signal Processing, vol. 44, $\mathrm{n}^{\circ}$ 12, 1996, p. 3017-3030.

[CAR 97] CARdoso J. F., «Infomax and Maximum Likelihood for Blind Source Separation », IEEE Signal Processing Letters, vol. 4, n 4, 1997, p. 112-114.

[CIC 02] Cichocki A., Amari S., Adaptative Blind Signal and Image Processing : Learning algoritms and applications, John Wiley, 2002.

[COM 94] Comon P., «Independent Component Analysis, a new concept ? », Signal Processing, vol. 36, $\mathrm{n}^{\circ} 3,1994$, p. 287-314.

[FLI 95] Fliess M., LÉvine J., MARTin P., Rouchon P., « Flatness and defect of non linear systems : Introductory theory and examples », Int J. Control, $\mathrm{n}^{\circ}$ 61, 1995, p. 1327-1361.

[GUI 80] GUIKHMAN I., SKOROKHOD A., Introduction à la théorie des processus aléatoires, Editions Mir, 1980.

[HYV 01] HyVARInen A., Karhunen J., OJa E., Independent Component Analysis, John Wiley, 2001.

[JAU 01] JAUlin J., KiefFer M., Didrit D., Walter E., Applied Interval Analysis, Springer, 2001.

[JAU 05] JAULIN L., Représentation d'état pour la modélisation et la commande des systèmes, Hermès - Lavoisier, 2005.

[J.L 97] J.L. LACOUme P. A., Comon P., Statistiques d'ordre supérieur pour le traitement du signal, Masson édition, 1997. 
18 Nom de la revue ou conférence (à définir par \submitted ou \toappear)

[JUT 91] Jutten C., HÉrault J., « Blind Separation of Sources, Part I : an adaptative Algorithm Based on Neuromimetic Architecture », IEEE Transactions on Signal Processing, vol. 24, 1991, p. 1-10.

[MOO 79] MOORE R., Methods and Applications of Interval Analysis, Society for Industrial and Applied Mathematics, Philadelphia, 1979.

[OLL 90] OLLIVIER F., «Le problème d' l'identifiabilité structurelle globale : approche théorique, méthodes effectives et bornes de complexité », $\mathrm{PhD}$ thesis, Ecole Polytechnique, 1990.

[PIC 89] PiCInbono B., Théorie des signaux et des systèmes, Dunod, 1989.

[WAL 97] WALTER E., PRONZATO L., Identification of parametric models from experimental data, Springer, 1997. 